\title{
Hightech macht Hightouch umso wichtiger - zur Neuentdeckung der Achtsamkeit
}

Daniel Hell

Korrespondenz:

Prof. Dr. med. Daniel Hell

Psychiatrische Universitätsklinik

Zürich

Lenggstrasse 31

CH-8032 Zürich
Kürzlich hat sich ein Kongress in Zürich mit dem Thema «Medizin an den Grenzen» beschäftigt. Dabei fiel mir die Aufgabe zu, die Grenzen der modernen Medizin mit Überlegungen zur Achtsamkeit in Zusammenhang zu bringen. Über Achtsamkeit zu reden und zu schreiben setzt aber voraus, dass man es achtsam tut. Dabei meint Achtsamkeit ja nicht einfach nur konzentriert sein, sondern impliziert auch, «mit vollem, ungeteilten Bewusstsein gegenwärtig zu sein». Gerade das war aber für mich schwierig. Ich brach mir nämlich in der Vorbereitungszeit für den Vortrag den Vorderarm und erlebte nicht nur ganz konkret eine fragmentierende Distraktion an meinem Körper, sondern wegen der aufkommenden Sorgen auch in meinem Denken und Fühlen. Abgelenkt-Sein und Zerstreuung sind aber bekanntlich das Gegenteil von Achtsamkeit. Das führt mich zu meiner ersten These. Insgesamt werde ich drei Thesen vertreten, die sowohl die Grenzen des Achtsamkeitspostulates wie die notwendige Ergänzung der technisch-wissenschaftlichen Medizin durch leib-seelische Achtsamkeit und Zuwendung (touch) zum Inhalt haben.

\section{Achtsamkeit ist ein Privileg}

Wer zu sehr in Sorge ist und wem das Leben übel mitspielt, wird sich nicht so leicht von irritierenden Gedanken und leidvollen Empfindungen frei machen können, um mit vollem, ungeteiltem Bewusstsein gegenwärtig zu sein.

In meinem beruflichen Alltag behandle ich manche Menschen mit rezidivierenden und mit chronifizierten Depressionen. Die meisten leiden auch daran, sich nicht selber aufmerksam und kritiklos wahrnehmen zu können. Von ihnen solches zu verlangen wäre blanker Zynismus. $\mathrm{Zu}$ offensichtlich ist die Behinderung, die eine schwere Depression mit sich bringt, weil diese Affektstörung die Konzentrationsfähigkeit und das Denkvermögen hemmt. Zu gross ist oft auch die Sorge, wie die Betroffenen angesichts ihrer depressiven Antriebs- und Entscheidungslosigkeit nur schon die nächsten Stunden überwinden können. Dennoch schliessen auch depressive Leiden vorsichtige Schritte hin zu grösserer Achtsamkeit nicht immer aus. Allerdings: Wer sich in schwerer Depression an Positivem fest-
Hightech et hightouch, ou la redécouverte de l'attention portée à la personne

Le développement technique et scientifique de la médecine a révolutionné les examens et le traitement de nombreuses maladies somatiques. Le triomphe de la médecine hightech repose sur un point de vue extérieur toujours plus perfectionné, à savoir sur ce qui est convenu d'appeler «la perspective de la troisième personne». La priorité donnée à l'approche objectivante a un revers: elle exige de l'être humain de se retrouver, de ne pas se perdre en tant que personne, donc en tant que sujet. Car il reste une distance entre ce que l'être humain éprouve physiquement, ce qu'il ressent affectivement et ce qu'il pense (selon la perspective de la première personne) et la considération extérieure de son corps en tant qu'objet. La redécouverte de l'attention portée à la personne est une tentative d'associer une perspective intérieure complémentaire à la perspective extérieure dominante, de compléter en quelque sorte la réification (hightech) par l'affection (hightouch).

halten möchte, findet keinen Ansatzpunkt. Er gleitet an reizlosen Wänden ab oder verliert sich in gähnender Langeweile. Wenn schon, bildet nicht das Erwünschte, sondern das Unerwünschte Widerhaken, an denen sich ein depressiver Mensch schmerzhaft spüren oder sogar festhalten kann.

Depression wird manchmal mit einem Sterben mitten im Leben verglichen. Tatsächlich ist sie eine Zeit des Abschiednehmens. Sie erfüllt keine Wünsche. Sie lässt vielmehr zurück, woran sich das Herz gebunden hat. Depression ist auch keine Zeit der grossen Gesten. Aber kleine Zeichen können wie beim Abschied wichtig werden: Eine versteckte Träne, eine kleine Aufmerksamkeit, 
ein verständnisvolles Schweigen. Es wird wichtig, was gesagt, aber auch was nicht gesagt wird. Es werden Dinge bedeutsam, die kein Gewicht haben und die schwere Reise nicht belasten. Denn Depression ist wie Abschied nehmen auch ein Aufbruch, wenn auch wider Willen und ohne bekanntes Ziel. Zuerst aber muss bewusst werden, was nicht mehr möglich ist. Erst dann kann die Schwere des Empfindens ein Eigengewicht bekommen und aus dem befremdenden Schicksal eine innere Erfahrung werden, eine bittere zwar, aber eine ureigene. Dieses neu gewonnene Innewerden (als Andeutung von Achtsamkeit) ist wie ein Geschenk, wie die Morgendämmerung oder der Regenbogen, der neues, eigenes Leben verspricht.

\section{Achtsamkeit ist kein Allheilmittel}

Streng genommen ist Achtsamkeit überhaupt kein Mittel, sondern ein Weg und ein Ziel. Der Unachtsame kann nicht das Mittel der Achtsamkeit einsetzen, um achtsam zu werden. Der spirituelle Begriff «Achtsamkeit» oder «mindfulness» (eine englische Übersetzung von «Sati» aus der Pali-Sprache) wurde zunächst vom buddhistischen Mönch Thich Nhat Hanh verwendet, um auszudrücken, was in der buddhistischen Lehre eine Art «unreflektiertes, reines Wahrnehmen» meint. Die ersten frühchristlichen Eremiten hatten Ähnliches im Sinn, wenn sie die «Herzensruhe» anstrebten, das meint ein unvoreingenommenes Wahrnehmen mit «reinem Herzen». Eine solche Art Achtsamkeit ist nicht funktionalisierbar. Als Mittel einsetzbar sind allenfalls Übungen, die es einem Menschen erleichtern können, dem Ziel der Achtsamkeit näher zu kommen, etwa Meditation oder Beten. Achtsames Innewerden kann auch gefördert werden, wenn sich ein Mensch dem Nächstliegenden zuwendet, z. B. dem eigenen Atmen, aber auch dem eigenen Gehen, Stehen oder Sitzen, überhaupt jeglichem leiblichen Tun. Hilfreich ist eine Umgebung, die Ruhe und Geborgenheit ausstrahlt. Achtsamkeit lässt sich aber nicht erzwingen - auch mit dem stärksten Willen nicht. Wer achtsam inne wird, reisst die Menschen und die Dinge nicht zu sich heran. Auch schneidet er die Welt nicht in Scheiben, um sie mit der Schärfe des Verstandes zu analysieren. Wer inne wird, erfährt vielmehr staunend, was geschieht.

Ich betone dies auch deshalb, weil es seit zwei Jahrzehnten einen spirituellen Therapieansatz zur Stressreduktion - die «mindfulness-based stress reduction» (MBSR) nach Jon Kabat-Zinn und seit einigen Jahren auch einen entsprechenden Ansatz zur Prophylaxe depressiver Episoden gibt - die «mindfulness-based cognitive therapy for depression» (MBCT) von Williams, Teasdale und Segal (diese wird seit kurzem auch an der PUK Zürich angewendet). Diese Therapieansätze verwenden Meditationspraktiken mit empirisch überprüftem Erfolg zur Stressreduktion und zur Depressionsprophylaxe und nehmen dafür den Begriff Achtsamkeit bzw. mindfulness für sich in Anspruch. Zweifelsohne haben diese Therapieansätze einen spirituellen Hintergrund und sind auch in diesem Kontext entstanden. Hingegen ist es meines Erachtens nicht angebracht, Achtsamkeit als Therapiemethode, gleichsam als funktionalisierbares Instrument, zu propagieren. Ein Achtsamsein mit reflektierter Absicht ist ein Widerspruch in sich selbst. Ich möchte deshalb am Beispiel der MBCT noch etwas genauer nachfragen, welchen Stellenwert Achtsamkeit in einer Behandlungssituation einnehmen kann.

In der Behandlung und in der Prävention von Depression spielt der Umgang mit negativen Gedanken (wie: ich bin nichts wert, ich habe keine Chance) eine wichtige Rolle. Wenn Menschen bereits früher depressive Episoden durchgemacht haben, können sich negative Gedanken, die für das depressive Erleben typisch sind, in ihnen stärker festgesetzt haben. Dadurch erhöht sich aber die Gefahr, dass im weiteren Verlauf bereits kurze, alltägliche Stimmungstiefs eine so starke Selbstinfragestellung auslösen, dass es nicht bei einem normalen Deprimiertsein über eine Enttäuschung bleibt, sondern sich (über Grübeln und Hadern) eine Stimmungsverschlechterung und schliesslich eine depressive Episode hochschaukelt. Es ist deshalb von prognostischer Bedeutung, dass Menschen mit einer erhöhten depressiven Rezidivgefahr im gesunden Intervall lernen, mit negativen Gedanken so umzugehen, dass sie sich nicht gleich mit ihnen identifizieren, sondern eine gewisse innere Distanz dazu finden. Das therapeutische Ziel besteht für diese Menschen also darin, auch bei einer Stimmungsverschlechterung die automatisch auftretenden negativen Gedanken nicht so persönlich zu nehmen, sondern als situationsbedingte, allenfalls flüchtige Phänomene, die nicht zu ihrem Personkern gehören, wahrzunehmen. Um eine solche Haltung gegenüber negativen Gedanken zu üben, werden in der MBCT Meditationsmethoden vermittelt, wie z.B. der Bodyscan oder die Atemmeditation. Je besser es dann depressiv gefährdeten Menschen gelingt, durch bewusstes, möglichst kritikloses Wahrnehmen eine gewisse Distanz zu den ihnen zufliegenden Gedanken einzunehmen - also sie gleichsam nicht vom Kopf in ihr Herz zu lassen, wie die Wüstenväter sagten -, umso geringer scheint das Rückfallrisiko zu sein. Erste empirische Ergebnisse zeigen 
nämlich, dass mit dieser Methode die Anzahl depressiver Rückfälle um die Hälfte gesenkt werden kann (in den vorliegenden Studien jedenfalls bei Kranken, die zuvor mindestens drei depressive Episoden erlebt hatten).

Persönlich bin ich allerdings der Auffassung, dass Achtsamkeit auch bei dieser Therapiemethode nicht als (symptomreduzierendes) Mittel eingesetzt wird und eine instrumentelle Funktion hat, sondern dass vertiefte Achtsamkeit überhaupt erst erfahren werden kann, wenn es den behandelten Menschen zuvor gelingt, sich von eigenen Befürchtungen und übertriebenen Erwartungen dank kognitiver Techniken und Meditationsübungen besser zu distanzieren. Dann aber dürfte Achtsamkeit mit Stimmungsstabilität parallel gehen.

Diese Präzisierung scheint mir insofern wichtig, als es nicht darum gehen kann, Achtsamkeit zu funktionalisieren und aus einer basalen Lebenshaltung bzw. einem spirituellen Anliegen eine kommerzialisierbare Technik zu machen.

Bezüglich der Frage, ob und wie spirituelle Ansätze in die Psychiatrie und Psychotherapie zu integrieren sind, beschäftigt mich besonders der Zusammenhang von Achtsamkeit und Schamgefühl. Scham stellt ein grundlegendes menschliches Selbstgefühl dar, ohne das personales Sein unmöglich ist. Das zeigt sich z. B. daran, dass Psychosekranke infolge der Fragmentierung ihres Selbst auch ihre Schamfähigkeit vorübergehend verlieren. Ist es aber möglich, diesem menschlichen Grundgefühl überhaupt achtsam - nicht nur aufmerksam - zu begegnen, oder ist es nicht vielmehr so, dass die Scham einen Menschen so vehement auf sich selbst zurückwirft, dass er sich unmittelbar zu verbergen sucht und gerade nicht mehr in der Lage ist, sich oder andern Menschen offen und absichtslos zu begegnen? Erfährt also die Achtsamkeit im Schamerleben ihre Grenzen, oder ist es sogar so, dass entwicklungspsychologisch (in der Kindheit) das Schamerleben überhaupt erst einen privaten, vor Fremden geschützten Raum im Menschen schafft, der schliesslich ein Selbstbewusstsein ermöglicht? Könnte aber Achtsamkeit - nach Ausbildung eines persönlichen Selbst - dazu beitragen, dass das eigene Ego wieder relativiert wird und der achtsame Mensch selbstbescheidener wird, auch weil er Schamerfahrungen besser akzeptieren kann und demzufolge sein Selbstgefühl nicht mehr in einer Ich-Jagd kompensatorisch - Scham verhüllend aufblähen muss?

Solche Fragen sind nicht bloss akademischer Art. Scham, auch Schamangst, ist das Gefühl, das meine Patientinnen und Patienten am meisten umtreibt und das mich in meinem Leben beson- ders intensiv beschäftigt hat. Es ist nicht damit getan - wie es viele, auch ich, versuchten -, Schamhaftigkeit mit Leistung zu kompensieren. Scham schafft sich so lange Raum, bis ein Mensch sich selber so annimmt, wie er nun einmal ist, also so lange, bis er sich nicht mehr zu verbergen braucht bzw. eine Enthüllung durch Dritte zu fürchten hat. Das aber heisst - altertümlich gesprochen -, «reinen Herzens» zu sein oder eine Art Schamstärke entwickelt zu haben.

\section{Die Neuentdeckung der Achtsamkeit in der Spätmoderne ist konsequent und notwendig}

Die Betonung der Achtsamkeit stellt eine Korrekturbewegung der neueren gesellschaftlichen Entwicklung dar, die sich - der Kürze halber - unter Schlagworten wie Objektivierung, Visualisierung und Digitalisierung (bzw. Virtualisierung) zusammenfassen lässt. Der Siegeszug der Moderne der im übrigen schon mit der scholastischen Betonung der Ratio und der Ablösung von der monastischen Kontemplation im Spätmittelalter begann - hat damit zu tun, dass das achtsame Innewerden als Erkenntnismethode durch überprüfbare, äussere Beobachtung und logische Durchdringung ersetzt wurde. Dieser Trend löste zwar immer Gegenbewegungen aus - man denke an die Mystik oder an die Romantik -, doch hat sich im Grossen und Ganzen die distanzierende und reflektierende Beobachtung auf Kosten des achtsamen Innewerdens durchgesetzt. Dabei ist der objektivierende Standpunkt der Moderne, der in der Naturwissenschaft seine Krönung erfährt, vom modernen Visualisierungsschub nicht zu trennen. Nicht nur die kopernikanische Wende in der Astronomie, sondern auch die Anwendung der Zentralperspektive in der Kunst hängt mit der technischen Vervollkommnung optischer Instrumente zusammen. So kam es, dass sich der abendländische Mensch in den letzten Jahrhunderten immer stärker als genau lokalisierbarer Körper im Raum wahrnahm - in der Medizin zuerst anatomisch, dann physiologisch und schliesslich molekularbiologisch. Demgegenüber büsste das akustische Zeiterleben mit dem narrativen Strang der Geschichte und von Geschichten an Bedeutung ein. Aber auch der taktil oder propriozeptiv empfindende Leib oder die sensorische Beheimatung in Geruchs- und Geschmacksempfindungen verloren an Bedeutung. Die Visualisierung obsiegte, damit aber auch die Objektivierung. Denn der Sehsinn eignet sich in spezifischer Weise, sich von etwas abzusetzen und etwas im Raum festzuhalten und zu objektivieren. Was wir sehen, dringt gleichsam weniger tief in uns ein, als was wir hören, fühlen, riechen. 
Es ist nicht von ungefähr, dass wir bei Verrat eher an einen Wortbruch, als inneren Vertrauensbruch, denken und dass Schizophreniekranke ihre Verfolger viel häufiger hören als sehen. Das genaue Hinsehen hilft sogar, sich von Sinnestäuschungen zu distanzieren, wie ganz allgemein der Sehsinn zur Distanzierung und Fixierung im Raume verhilft. Deshalb fühlen sich manche Menschen auch durch stechende Blicke anderer Personen wie enthumanisiert und $\mathrm{zu}$ blossen Objekten gemacht, was subjektiv wiederum als besonders entwertend und beschämend erlebt werden kann.

Dass die Visualisierung und damit zusammenhängend eine Art Körperkult unser Leben immer mehr bestimmen, zeigt sich auch in der Medizin. Hier hat die Objektivierung, die Verdinglichung unseres Lebens, vielleicht ihre grössten Triumphe errungen. Man denke nur an die revolutionär verbesserten Behandlungsmöglichkeiten von Infektions- oder Herzkranzkrankheiten oder an die extrem verfeinerten Operationstechniken an fast allen menschlichen Organen.

An diesem Fortschritt möchte auch mein Fachgebiet teilhaben. Das ist bisher aber nur teilweise gelungen. Wenn man etwa den medikamentösen Fortschritt der Behandlung von Herz- und Kreislauferkrankungen in den letzten 30 Jahren mit dem vergleichsweise geringen psychopharmakologischen Fortschritt im gleichen Zeitraum vergleicht, so ist der Unterschied frappant. Der Grund scheint mir darin zu liegen, dass psychische Störungen sehr viel stärker als primär organische Erkrankungen mit dem inneren Erleben und mit persönlichen Einstellungen zu tun haben, also genau damit, was auch mit fortgeschrittensten neuroradiologischen Methoden nicht so leicht visualisierbar ist. Man kann dagegen einwenden, dass die Neurowissenschaft unser Wissen um zentralnervöse Vorgänge dank (funktioneller) neuroradiologischer Verfahren und molekularbiologischen Methoden in früher unvorstellbarer Weise ausgeweitet hat. Aber gerade dieser Umstand gibt zu denken. Die enormen neurophysiologischen und physikalischchemischen Erkenntnisfortschritte konnten bisher kaum zur effizienten Behandlung psychischer Störungen umgesetzt werden. Zwischen Neurochemie und Neurophysiologie des Gehirns einerseits und psychischem Erleben ist ein Sprung zu überwinden, der gleichzeitig einen Perspektivenwechsel erfordert: Hier der objektivierende Blick von aussen, die Perspektive der dritten Person, dort das subjektive Erleben aus erster Hand, die Perspektive der ersten Person. Ein solcher Sprung findet sich weniger bei somatischen und neurologischen Störungen.
Und Hand aufs Herz: Wissen wir so genau, was in digital verarbeitenden Computersimulationen (etwa im fMRI des Gehirns eines depressiven Menschen) abgebildet ist? Friedrich Dürrenmatt hat sich vor vielen Jahren einmal gefragt, inwieweit der Normalbürger versteht, was geschehe, wenn er ein elektrisches Gerät an den Stecker anschliesse. Heute hat die Computerwissenschaft sich noch einiges weiter vom Alltagsverständnis der Menschen entfernt. Wir bewegen uns auch in der Medizin auf eine zunehmend virtuelle Welt hin, denn was wir als fMRI-Bilder vor uns haben, ist keine natürliche Wahrnehmung, sondern eine hochverrechnete Digitalisierung bestimmter hirnorganischer Vorgänge wie Blutfluss- oder Glukosekonzentrationsveränderungen.

Natürlich kann diese Technik von Experten verstanden und adäquat interpretiert werden. Und natürlich sind solche Resultate nicht nur faszinierend, sondern für das Studium organischer Hirnvorgänge auch fundamental. Das ist hier nicht der Punkt. Worauf ich hinauswill, ist der einfache, aber grundlegende Umstand, dass wir uns mit der Anwendung dieser Techniken noch weiter von uns selbst entfernen. Wir erhalten ein Bild von uns, das etwas grundsätzlich anderes ist, als was wir innerlich erleben. Max Frisch formulierte in seinen Tagebüchern einmal knapp und präzis: «Denn das Fremdeste, was man erfahren kann, ist das Eigene einmal von aussen gesehen.» Die Grenze der technisch-wissenschaftlichen Medizin liegt weniger in ihrer Entwicklungsfähigkeit, als in ihrem Ansatz. Sie bedarf einer Ergänzung.

Gerade auch die faszinierenden neurowissenschaftlichen Fortschritte zwingen uns dazu, uns selbst neu zu finden. Indem sie uns aus der Perspektive der dritten Person - von aussen - immer genauer sehen lassen, sind wir darauf angewiesen, uns als Personen, als Subjekte nicht zu verlieren. Wir sind, was wir sind, weil wir das, was sich von aussen an uns wahrnehmen lässt, mit unserem eigenen, inneren Leben verbinden können. So sind wir Objekt und Subjekt zugleich. Wir haben einen Körper, den wir wie ein Objekt oder wie ein Instrument einsetzen können. Wir sind aber zugleich Leib, der uns fühlen und spüren lässt, wie uns zumute ist. Würden wir unser Erleben nur als Epiphänomen betrachten und alles auf die Karte der objektivierenden Aussensicht setzen, gingen wir uns selbst verloren. Deshalb ist die Neuentdeckung der Achtsamkeit in der heutigen Zeit nur konsequent und notwendig. Je stärker der Druck von aussen wird, desto wichtiger wird auch die Stärkung unseres inneren Erlebens. 